\title{
Relações entre exercício físico outdoor e indoor, bem-estar subjetivo e conexão com a Natureza
}

\section{Humberto da Nóbrega Alves ${ }^{1, *}$, Luane Maria Melo Azeredo $^{2}$, Luiz Carlos Serrano López $^{1}$ e Reinaldo Farias Paiva de Lucena ${ }^{1}$}

${ }^{1}$ Universidade Federal da Paraíba. Centro de Ciências Exatas e da Natureza. Programa de Pós-Graduação em Desenvolvimento e Meio Ambiente. Campus I. João Pessoa-PB, Brasil (CEP 58051-900).*E-mail: humbertonobrega@yahoo.com.br.

${ }^{2}$ Universidade Federal Rural de Pernambuco. Centro de Ciências Biológicas. Programa de Pós-Graduação em Etnobiologia e Conservação da Natureza. RecifePE, Brasil (CEP 52171-900).

Resumo. 0 objetivo da pesquisa foi investigar as relações entre conexão com a Natureza, bem-estar subjetivo e saúde de praticantes de exercícios físicos outdoor e indoor no Município de João Pessoa/PB. Foram analisados dados socioeconômicos, de composição corporal, de conexão com a Natureza, de bem estar subjetivo (emoções positivas, negativas e experiência subjetiva com o exercício em uma amostra de 300 praticantes de exercícios outdoor e indoor. Foi utilizado o Teste de Correlação de Sperman e o Teste t para comparação das variáveis do estudo com p $<0,005$ e $95 \%$ de intervalo de confiança. Foram observadas diferenças significativas pelos Teste $\mathrm{T}$ e Teste de Correlação de Spearman entre o grupo outdoor e grupo indoor com a RCQ percentagem de gordura corporal, somatório das dobras cutâneas, fadiga, bem-estar, afetos positivos, afetos negativos e conexão com a Natureza. Também foram observadas dentro do grupo outdoor (grupo praça e grupo praia) diferenças significativas no IMC e afetos negativos. Pelo Teste de Spearman observou-se correlações negativas entre aflição e bem-estar, fadiga e bem-estar, afetos negativos e conexão com a Natureza, afetos negativos e afetos positivos, RCQ e IMC, dobras cutâneas e RCQ; já as correlações positivas foram entre fadiga e aflição, afetos positivos e bem-estar, afetos negativos e aflição, afetos negativos e fadiga, IMC e aflição, dobras cutâneas e IMC. Estes resultados demonstram as relações existentes entre exercício outdoor/indoor, meio ambiente, bem estar e saúde, sendo um parâmetro importante para o fortalecimento e ampliação dos programas de treinamento com exercícios físicos para a melhoria e manutenção da saúde e qualidade de vida da população.

Palavras-chave: Exercício; Qualidade de vida; Saúde; Meio ambiente.
Recebido

13/10/2019

Aceito

22/12/2019

Publicado

31/12/2019

Acesso aberto

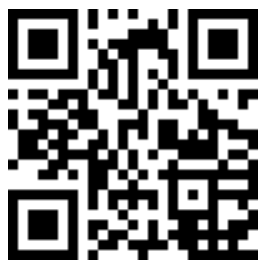

ORCID

(D) 0000-0002-3081-537X Humberto da Nóbrega Alves

(D) 0000-0003-2944-6439 Luane Maria Melo de Azeredo

(D) 0000-0002-9983-5563

Luiz Carlos Serrano

López

(ㄷ) 0000-0003-4775-7775 Reinaldo Farias Paiva de Lucena 


\begin{abstract}
Relations between physical exercise outdoor and indoor, subjective well-being and connectedness to nature. The aim of this research was to investigate the relations between nature connections, subjective well-being and health of indoor and outdoor exercises practitioners in João Pessoa, Paraiba, Brazil. Socioeconomic data, body composition, nature connection, subjective well-being (positive and negative emotions) and subjective experience with exercises were analyzed in a sample of 300 practitioners of outdoor and indoor exercises. The Sperman correlation and Mann-Whitney test were used for comparison of the study variables with $\mathrm{p}<0.005$ e $95 \%$ of confidence interval. Significative differences were observed by $t$ test and spearman correlation test between the outdoor and indoor groups with the WHR, percentage of body fat, sum of skinfolds, fatigue, well-being, positive affects, negative affects and nature connection. It was also observed within the outdoor group (plaza group and beach group) significative differences in BMI and negative effects. By the Spearman test it was observed negative correlations between affliction and well-being, fatigue and well-being, negative affects and nature connection, negative affects and positive affects, WHR and BMI, skinfolds and WHR; the positive correlations were between fatigue and affliction, positive affects and well-being, native affects and affliction, negative affect and fatigue, BMI and affliction, skinfolds and BMI. These results demonstrate the existing relations between indoor/outdoor exercise, environment, well-being and health as a important parameter for the strengthening and ampliation of training programs with exercises to improvement and maintenance of health and quality of life of the population.
\end{abstract}

Keywords: Exercise; Quality of life; Health; Environment.

\section{Introdução}

Estudos recentes tem apontado para interconexões importantes entre a prática regular de exercício físico (outdoor e indoor), bem-estar subjetivo e conexão com a natureza (Calogiuri et al., 2005; Matsouka et al., 2005; Loureiro e Veloso, 2014; Pasanen et al.,2014; Puett et al., 2014; Weng e Chiang, 2014; Lacharité-Lemieux e Dionne, 2016; Rogerson et al., 2016; Wood et al., 2016;Krinski et al., 2017; Niedermeier et al., 2017; Fuegen e Breitenbecher, 2018; Legrand et al., 2018; Alves et al., 2019),podendo ser considerada como uma ferramenta importante na melhoria da saúde e qualidade de vida das pessoas, assim como no combate aos danos provocados pelo sedentarismo e também na incorporação de hábitos saudáveis associados ao exercício físico (Minayo et al., 2000; Carvalho, 2001; Piko e Keresztes, 2006; Haskell et al., 2007).

Bem-estar pode assim ser entendido como a integração harmoniosa entre os componentes mentais, físicos, espirituais e emocionais (Nahas et al., 2000), sendo o bem-estar como um dos conceitos chaves de saúde, destacando o bem-estar subjetivo, bem-estar psicológico e bem-estar no trabalho (Siqueira e Padovan, 2008) e um dos conceitos bastante estudados por outros pesquisadores da área de saúde (Diener et al., 2003; Albuquerque e Tróccoli, 2004).

Em alguns estudos pesquisadores tem demarcado a existência de relação entre treinamento com o exercício físico 
outdoor/indoor com o bem-estar, com a saúde, assim como com o meio ambiente (Florindo et al., 2011; Silva et al., 2014), sendo observado em alguns estudos que a prática do exercício físico outdoor (exercício ao ar livre) tem sido associada a melhorias de indicadores de bem-estar subjetivo e de conectividade com a natureza (Loureiro e Veloso, 2014; Weng e Chiang, 2014; Pasanen et al.,2014; Puett et al., 2014; Wood et al., 2016; Calogiuri et al., 2105; Rogerson et al., 2016; Krinski et al.,2017; Niedermeier et al., 2017; Fuegen e Breitenbecher, 2018), encontrando associação direta entre o exercício outdoor com melhorias no aumento do foco, atenção, afetos positivos, sensação de prazer, humor, energia, adesão ao exercício e diminuição da fadiga.

Vale salientar também relações positivas de conexão dos exercícios físicos com o meio ambiente, mais especificamente em relação ás questões ambientais de preservação e conservação da Natureza (Florindo et al., 2011; Silva et al., 2014; Marselle et al., 2014), sendo que a conectividade com o meio ambiente pode ser considerada como um preditor de bem-estar subjetivo e de saúde geral (Loureiro e Veloso, 2014).

Estudos envolvendo exercícios físicos outdoor, indoor e bem-estar tem apontado claramente para ganhos significativos maiores na prática de exercícios com treinamentos outdoor do que nos treinamentos indoors, indicando que os praticantes de exercícios outdoor apresentaram melhorias estatisticamente significativas na melhoria do bem estar psicológico e diminuição dos afetos negativos (Matsouka et al., 2005; Loureiro e Veloso, 2014; Puett et al., 2014), na melhoria do humor (Wood et al., 2016; Niedermeier et al., 2017; Fuegen e Breitenbecher, 2018) e na melhoria da atenção (Weng e Chiang, 2014; Rogerson et al., 2016; Fuegen e Breitenbecher, 2018).
Assim, este artigo tem como objetivo avaliar as relações entre exercício físico (outdoor e indoor), bemestar subjetivo e conexão com a natureza em uma população adulta urbana, levando em consideração nesta análise as características socioeconômicas (idade, sexo, renda, nível de escolaridade), identificação de hábitos de exercício físico e de repouso noturno, fisiológica/ composição corporal (Índice de Massa Corporal, Relação Cintura Quadril e Dobras Cutâneas), conexão com a natureza (perfil e comportamentos pro ambientais), psicoemocionais (emoções positivas e negativas) e percepção subjetiva com o exercício físico.

\section{Métodos}

Foi realizado um estudo quantitativo com caráter descritivo, analítico e interpretativo, de outubro de 2017 a janeiro de 2018, no Município de João Pessoa, envolvendo um grupo amostral de 300 pessoas com idade entre 18 e 80 anos, selecionados de acordo com uma análise estatística populacional das cinco zonas da cidade (norte, sul, leste, oeste e centro da cidade), distribuídos no grupo de praticantes de exercícios físicos indoors $(\mathrm{n}=150)$ que eram praticantes de musculação, ginástica aeróbica/localizada e natação de sete academias de ginástica previamente selecionadas pelo zoneamento da cidade citado anteriormente, considerando as academias de ginástica como os espaços indoors (ambientes fechados) e no Grupo de praticantes de exercícios físicos outdoor $(n=150)$ que eram praticantes de caminhada, corrida e ciclismo, também previamente selecionadas pelo zoneamento das cinco áreas da cidade citadas anteriormente, considerando praças públicas e praias como os espaços outdoor (ambiente ao ar livre).

Recorreu-se as técnicas exploratórias de coleta de dados por 
meio de cinco instrumentos de coleta de dados:

$\begin{array}{r}\text { Formulário } \\ \text { de }\end{array}$
avaliação
instrumento de análise do perfil
socioeconômico (sexo, idade,
escolaridade, rendimento salarial e
condições de trabalho), considerando os
indicadores sociais como medidas
dotadas de um significado social
substantivo para cruzamento com outras
variáveis de estudo (Graciano, 2013).

Formulário de avaliação da composição corporal: analisou-se neste instrumento o Índice de Massa Corporal, Relação Cintura Quadril e Dobras Cutâneas. Considerando a avaliação da composição corporal como um importante elemento da aptidão física, devido à existência das relações entre a quantidade e a distribuição de gordura corporal com alterações no nível de aptidão física, bem como no quadro de saúde das pessoas (Garcez, 2017). Para as análises do Índice de Massa Corporal (IMC) e da Relação Cintura Quadril (RCQ) foram utilizadas as classificações de Garrow (1983) e Dehoog (1998), respectivamente. Para as dobras cutâneas foi empregada a tabela apresentada por Guedes (1994) para estimar o cálculo de percentual de gordura através das dobras cutâneas e a classificação do percentual de gordura pelos protocolos de Pollock e Wilmore (1993) e a de Petroski (1999).

\section{Escala de conexão com a Natureza: investigou-se neste} instrumento a conectividade com o natureza, sendo esta variável importante elemento da determinação de comportamentos humanos frente ao meio ambiente, seja ele natural ou construído, tendo importantes implicações em atitudes e comportamentos pró-ambientais e consequentemente, na qualidade de vida das gerações presentes e futuras ${ }^{33}$, sendo utilizado para esta análise a escala de
Mayer e Franz (2004), já utilizada também em outros estudos (Pessoa, 2016).

Escala de emoções positivas e negativas (PANAS): instrumento de verificação das emoções positivas e negativas relacionadas ao bem-estar subjetivo para compreender a relação entre bem-estar subjetivo e exercícios físicos, utilizando-se da escala de Watson e Clark (1994), categorizando o total de 20 emoções da escala de dimensões gerais a partir de palavras e frases que descreveram sentimentos e emoções que os pesquisados sentiram durante a última semana anterior a aplicação deste instrumento de pesquisa), sendo 10 positivas (ativo, alerta, atento, determinado, entusiasmado, empolgado, inspirado, interessado, orgulhoso e forte) e 10 negativas (com medo, apavorado, nervoso, inquieto, irritável, hostil, culpado, envergonhado, chateado e aflito).

\section{Escala de experiência subjetiva} com exercício: teve como objetivo investigar a relação entre experiência subjetiva de bem-estar e exercício físico, buscando assim avaliar a percepção sobre a experiência subjetiva com exercício, além de ter dado subsídios importantes para a compreensão da relação entre bem-estar subjetivo e exercícios físicos, sendo uma escala mais específica de experiência subjetiva com o exercício, indicando a forma como os sujeitos pesquisados se sentem normalmente após a prática do exercício físico. $\mathrm{Na}$ escala os itens são resumidos para criar um escore sucinto de bemestar positivo (itens 1, 4, 7, 10), aflição psicológica (itens $2,5,8,11$ ) e fadiga (itens 3, 6, 9, 12) (Cabral e Palmeira, 2003).

Esta pesquisa foi submetida à avaliação do Comitê de Ética da Universidade Federal da Paraíba, em 28/09/2017 (Parecer no 2.304.048A), sendo todos os instrumentos avaliados em 27/08/2017 (Processo CAAE no 
70957617.0.0000.5188), tendo sido aprovado com recomendação para aplicação aos sujeitos pesquisados de acordo com a Resolução no 466/2012, referentes às diretrizes e normas regulamentadoras de pesquisa envolvendo seres humanos do Conselho Nacional de Saúde (Brasil, 2012).

Antes da aplicação de todos os instrumentos acima citados foi apresentado a todos os sujeitos pesquisados o Termo de Consentimento Livre e Esclarecido (TCLE) para o consentimento dos mesmos, sendo também apresentado aos responsáveis pelas instituições que foram realizadas as pesquisas o termo de anuência, ambos os termos de acordo com a Resolução $\mathrm{n}^{-}$ $466 / 2012$ referentes às diretrizes e normas regulamentadoras de pesquisa envolvendo seres humanos do Conselho Nacional de Saúde (Brasil, 2012).

\section{Estatística}

Os dados foram analisados através de teste de comparação de medianas (Mann-Whitney), para comparar os resultados das variáveis de bem-estar, conexão com a natureza e afetos entre os grupos de praticantes de exercícios indoor e outdoor. Utilizou-se também uma matriz de Correlação de Spearman para correlacionar as variáveis que mediam afetos, bem-estar, conexão com a natureza e medidas de saúde entre si e o Teste de Mann-Whitney comparando as variáveis do grupo outdoor $\mathrm{x}$ indoor e do entre grupo indoor praça $x$ grupo indoor praias. Além disso, os dados socioeconômicos dos participantes foram tabelados em porcentagem para melhor traçar o perfil dos grupos indoors e outdoors, além de também realizar a análise das medias e desvios padrões. As análises foram feitas no Programa
Estatístico R (versão 3.4.1) e o intervalo de confiança adotado foi de $95 \%$.

\section{Resultados}

\section{Dados socioeconômicos}

0 presente estudo foi realizado com 300 pessoas com faixa etária entre 18 e 80 anos, sendo com 131 pessoas do sexo masculino correspondendo a $44 \%$ com idade média e desvio padrão de $36,31 \pm 14,77$ e 169 pessoas do sexo feminino correspondendo a $56 \%$, com idade média e desvio padrão de $40,81 \pm 15,71$.

Em relação a escolaridade observou-se que a maioria dos homens tinham curso superior completo, sendo no Grupo Outdoor o percentual de 32\% e no grupo indoor o percentual de $29 \%$; já as mulheres na sua maioria tinham o ensino médio completo, sendo no Grupo Outdoor o percentual de $28 \%$ e no grupo indoor o percentual de $26 \%$, como pode ser visualizado na Tabela 1 referente a distribuição percentual da população da pesquisa de campo segundo as características socioeconômicas da pesquisa.

Em termos de rendimento salarial, sem considerar $o$ quantitativo dos salários recebidos, observou-se que a maioria dos sujeitos da pesquisa tinham rendimento salarial acima de um salário mínimo. Referenciando-se a distribuição percentual a população da pesquisa de campo, segundo as caracteristicas socioeconômicas da pesquisa, os resultados podem ser visualizados na Tabela 1, destacando-se que em média $60 \%$ dos homens e mulheres de ambos os grupos (outdoor e indoor) praticantes de atividades físicas ganham acima de um salário mínimo. 
Tabela 1. Distribuição percentual da população da pesquisa de campo segundo as características socioeconômicas realizada em João Pessoa/PB 2017/2018.

\begin{tabular}{|c|c|c|c|c|c|}
\hline \multirow{2}{*}{$\begin{array}{l}\text { Características } \\
\text { socioeconômicas }\end{array}$} & \multirow[b]{2}{*}{ Categorias } & \multicolumn{2}{|c|}{ Outdoor $(n=150)$} & \multicolumn{2}{|c|}{ Indoor $(n=150)$} \\
\hline & & $\begin{array}{c}\text { Masculino } \\
(n=62) \%\end{array}$ & $\begin{array}{l}\text { Feminino } \\
(n=88) \%\end{array}$ & $\begin{array}{c}\text { Masculino } \\
(n=69) \%\end{array}$ & $\begin{array}{c}\text { Feminino } \\
(n=81)\end{array}$ \\
\hline \multirow{10}{*}{ Escolaridade } & Não Alfabetizado & - & 1 & - & - \\
\hline & Somente alfabetizado/a & 3 & 2 & - & 1 \\
\hline & Ensino fundamental incompleto & 10 & 14 & 4 & 1 \\
\hline & Ensino Fundamental completo & 8 & 7 & - & 3 \\
\hline & Ensino médio incompleto & 8 & 2 & 7 & 9 \\
\hline & Ensino médio completo & 24 & 28 & 22 & 26 \\
\hline & Ensino superior incompleto & 10 & 7 & 26 & 25 \\
\hline & Ensino superior completo & 32 & 26 & 29 & 24 \\
\hline & Pós graduação incompleta & 2 & 5 & 6 & 4 \\
\hline & Pós graduação completa & 3 & 8 & 6 & 9 \\
\hline \multirow{4}{*}{ Rendimento salarial } & Sem rendimento & 16 & 20 & 20 & 18 \\
\hline & Menos de um salário mínimo & 7 & 6 & 2 & 2 \\
\hline & um salário mínimo & 13 & 14 & 16 & 16 \\
\hline & Mais de um salário mínimo & 64 & 60 & 62 & 64 \\
\hline \multirow{4}{*}{$\begin{array}{l}\text { Condições de } \\
\text { trabalho }\end{array}$} & Remunerado & 65 & 55 & 80 & 78 \\
\hline & Não remunerado & 19 & 20 & 20 & 18 \\
\hline & $\begin{array}{l}\text { Trabalho na produção para } \\
\text { consumo ou uso próprio }\end{array}$ & 2 & 2 & - & - \\
\hline & Aposentado/pensionista & 14 & 23 & - & 4 \\
\hline
\end{tabular}

Com relação às condições de trabalho dos sujeitos pesquisados observou-se que a maioria eram remunerados, Tabela 1. Em ambos os grupos entrevistados os homens $(65 \%$ outdoor e 80\% indoor) disseram ser melhor remunerados do que as mulheres (55\% outdoor e $78 \%$ indoor).

\section{Composição corporal}

Em se tratando da composição corporal, avaliando o Índice de Massa Corporal não se observou diferenças significativas pelo teste de MannWhitney entre o Grupo Outdoor $(25 \pm 5,40)$ e o Grupo Indoor $(25 \pm 6,43)$, com $\mathrm{W}=10823$ e $\mathrm{p}=0,57$ para esta variável; Considerando-se a Relação Cintura Quadril (RCQ) também não se observou diferenças significativas entre o Grupo Outdoor $(2 \pm 1)$ e Grupo Indoor $(2 \pm 1)$, com valores de $W=11475$, e $p=0,7$; por outro lado, em relação ao somatório das dobras cutâneas, observou-se diferenças significativas entre o Grupo Outdoor (59 \pm 22$)$ e Grupo Indoor (54 \pm 23$)$, com valores de
$\mathrm{W}=85005$ e $\mathrm{p} \leq 0,001$ para esta variável, sendo assim também significativo as diferenças do percentual de gordura corporal das dobras cutâneas entre o Grupo Outdoor $(23 \pm 7,18)$ e Grupo Indoor $(22 \pm 8,61)$, com valores de $W=86855$ e $p$ $\leq 0,005$ para esta variável, como pode ser visualizado na tabela 2 com dados desta variável dos sujeitos da pesquisa.

\section{Bem estar subjetivo}

Em relação ao bem estar subjetivo pela percepção da experiência subjetiva com exercício no tocante a fadiga, observou-se diferenças significativas pelo teste de MannWhitney entre o Grupo Outdoor $(2,5 \pm 1,5)$ e o Grupo Indoor $(3,0 \pm 2)$, com valores de $\mathrm{W}=15062$, e $\mathrm{p}<0,001$ para esta variável; em relação a aflição ou mal estar psicológico não se observou diferenças significativas entre o Grupo Outdoor $(1 \pm 0,25)$ e o Grupo Indoor $(1 \pm 0,25)$, com valores de $W=10824$ e $\mathrm{p}=0,49$; em relação ao bem estar observou-se diferenças significativas entre o Grupo Outdoor $(6 \pm 1,25)$ e o Grupo Indoor $(5 \pm 1,68)$, com valores de 
$\mathrm{W}=7241,5$ e p $<0,001$, como pode ser visualizado na tabela 2 com dados desta variável dos sujeitos da pesquisa.

Em relação ao bem estar subjetivo pelos afetos (emoções) positivos observou diferenças significativas pelo Teste de MannWhitney entre o Grupo Outdoor $(3 \pm 0,5)$ e o Grupo Indoor $(3 \pm 0,67)$, com valores de $\mathrm{W}=73790$ e $\mathrm{p}<0,001$ para esta variável; também encontrando diferenças significativas para os afetos (emoções) negativos entre o Grupo Outdoor $(2 \pm 0,9)$ e o Grupo Indoor $(2 \pm 0,8)$, com valores de
$\mathrm{W}=13216$ e $\mathrm{p}<0,005$, como pode ser visualizado na Tabela 2 com dados desta variável dos sujeitos da pesquisa.

\section{Conexão com a natureza}

Em relação a conexão com a natureza observou diferenças significativas pelo Teste de MannWhitney entre o Grupo Outdoor $(3,57 \pm 0,5)$ e o Grupo Indoor $(3,35 \pm 0,98)$, com valores de $\mathrm{W}=64880$ e $\mathrm{p}<0,001$ para esta variável, como pode ser visualizado na tabela 2 com dados desta variável dos sujeitos da pesquisa.

Tabela 2. Medianas, intervalos interquartílicos e valores do Teste de Mann-Whitney de comparações entre as variáveis de composição corporal, bem estar subjetivo (experiência subjetiva com exercício e emoções positivas/emoções negativas) e conexão com a natureza dos entrevistados na pesquisa de campo $(\mathrm{N}=300)$ referentes ao grupo outdoor* $(\mathrm{n}=150)$ e ao grupo indoor** $(\mathrm{n}=150)$, realizada em João Pessoa 2017/2018 (N=300).

\begin{tabular}{|c|c|c|c|c|c|}
\hline Variáveis da pesquisa & $\begin{array}{l}\text { Outdoor* } \\
\text { Md } \pm \text { IIR*** }\end{array}$ & $\begin{array}{c}\text { Indoor** } \\
\text { Md }_{\text {dIIR*** }}\end{array}$ & $\mathbf{W}$ & $\mathbf{p}$ & $\begin{array}{c}\text { Significativo } \\
(p<0,05)\end{array}$ \\
\hline $\begin{array}{l}\text { Índice de Massa Corporal } \\
\text { (IMC) }\end{array}$ & $25 \pm 5,40$ & $25 \pm 6,43$ & 10823 & 0,570 & Não \\
\hline $\begin{array}{l}\text { Relação Cintura Quadril } \\
\text { (RCQ) }\end{array}$ & $2 \pm 1$ & $2 \pm 1$ & 11475 & 0,700 & Não \\
\hline $\begin{array}{l}\text { Somatório das dobras } \\
\text { cutâneas } * * * *\end{array}$ & $59 \pm 22$ & $54 \pm 23$ & 85005 & $<0,001$ & Sim \\
\hline $\begin{array}{l}\text { Percentual de gordura } \\
\text { corporal das dobras } \\
\text { cutâneas } * * * *\end{array}$ & $23 \pm 7,18$ & $22 \pm 8,61$ & 86855 & $<0,005$ & Sim \\
\hline Fadiga & $3 \pm 1,5$ & $3 \pm 2$ & 15062 & $<0,001$ & Sim \\
\hline Aflição & $1 \pm 0,25$ & $1 \pm 0,25$ & 10824 & 0,490 & Não \\
\hline Bem estar & $6 \pm 1,25$ & $5 \pm 1,68$ & 72415 & $<0,001$ & Sim \\
\hline Afetos positivos & $3 \pm 0,5$ & $3 \pm 0,67$ & 73790 & $<0,001$ & Sim \\
\hline Afetos negativos & $2 \pm 0,9$ & $2 \pm 0,8$ & 13216 & $<0,005$ & Sim \\
\hline $\begin{array}{l}\text { Conexão com a natureza } \\
(\mathrm{CN})\end{array}$ & $3,57 \pm 0,5$ & $3,35 \pm 0,98$ & 64880 & $<0,001$ & Sim \\
\hline
\end{tabular}

Legenda: *Busto de Tamandaré (Praia de Tambaú-Cabo Branco), Praça General Lavanery Wanderley, Sport \& Saúde Academia, Corpo livre Academia, Parque da Lagoa Solon de Lucena, Praça da Paz; Praça do Coqueiral e Entorno do Almeidão/Ronaldão; **RED Zone Academia-CrossFit Monster Factory, Bio Forma Academia, Pro Life Academia, Fórmula Fitness Academia e G+ Academia; *** $M_{d}$ (Mediana) $\pm I I R$ (intervalo interquartílico) / **** dobras cutâneas tricipital, supra ilíaca e abdominal (homens) e dobras cutâneas da coxa, supra ilíaca e subescapular (mulheres).

Além da comparação entre o Grupo Outdoor e Grupo Indoor, também foi feito o teste de Mann-Whitney de comparações entre as variáveis da pesquisa dentro do Grupo Outdoor, comparando os resultados dos sujeitos pesquisados nas praias de Tambaú/Cabo
Branco e sujeitos pesquisados nas praças do projeto "João Pessoa, vida saudável" (Praça General Lavanery Wanderley, Praça da Paz, Praça do Coqueiral, Entorno do Almeidão/Ronaldão e Parque da Lagoa Solon de Lucena), que promovem atividades físicas regulares ao 
ar livre, pelo menos três vezes por semana. Diante do teste de MannWhitney com estes indivíduos observou diferenças significativas apenas com os afetos (emoções) negativos entre 0 Grupo Outdoor das praças $(1,9 \pm 0,7)$ e o
Grupo Outdoor das praias $(1,6 \pm 0,7)$, com valores de $W=2939,5$ e $p<0,001$, como pode ser visualizado na tabela $3 \mathrm{com}$ dados desta variável dos sujeitos da pesquisa.

Tabela 3. Medianas, intervalos interquartílicos e valores do Teste de Mann-Whitney de comparações entre as variáveis de composição corporal, bem estar subjetivo (experiência subjetiva com exercício e emoções positivas/emoções negativas) e conexão com a natureza dos entrevistados na pesquisa de campo $(N=300)$ referentes realizada em praças* $(n=110)$ e praias** $(n=40)$ da cidade de João Pessoa 2017/2018.

\begin{tabular}{|c|c|c|c|c|c|}
\hline $\begin{array}{l}\text { Variáveis da } \\
\text { pesquisa }\end{array}$ & $\begin{array}{c}\text { Outdoor } \\
\text { Praças* } \\
\text { Md } \pm \text { IIR*** }\end{array}$ & $\begin{array}{l}\text { Outdoor } \\
\text { Praias** } \\
\text { Md } \pm \text { IIR }^{* * *}\end{array}$ & $\mathbf{w}$ & $\mathbf{p}$ & $\begin{array}{c}\text { Significativo } \\
(p<0,05)\end{array}$ \\
\hline $\begin{array}{l}\text { Índice de Massa } \\
\text { Corporal (IMC) }\end{array}$ & $25,53 \pm 5,91$ & $24,64 \pm 4,83$ & 2610,5 & 0,08 & Não \\
\hline $\begin{array}{c}\text { Relação Cintura } \\
\text { Quadril (RCQ) }\end{array}$ & $2 \pm 1$ & $2 \pm 0,25$ & 1970 & 0,22 & Não \\
\hline $\begin{array}{c}\text { Percentual de gordura } \\
\text { corporal das dobras } \\
\text { cutâneas }\end{array}$ & $23 \pm 7,6$ & $23,4 \pm 5,86$ & 2302,5 & 0,66 & Não \\
\hline $\begin{array}{c}\text { Somatório das dobras } \\
\text { cutâneas }\end{array}$ & $58,5 \pm 23$ & $60,5 \pm 19,5$ & 22,64 & 0,78 & Não \\
\hline Fadiga & $2,5 \pm 1,43$ & $2,25 \pm 1,31$ & 2065 & 0,56 & Não \\
\hline Aflição & $1 \pm 0,25$ & $1 \pm 0,5$ & 1984 & 0,28 & Não \\
\hline Bem estar & $5,75 \pm 1,25$ & $5,62 \pm 1,06$ & 2355 & 0,50 & Não \\
\hline Afetos positivos & $3,5 \pm 0,47$ & $3,35 \pm 0,6$ & 2523 & 0,16 & Não \\
\hline Afetos negativos & $1,9 \pm 0,7$ & $1,6 \pm 0,7$ & 2939,5 & $\mathrm{p}<0,001$ & Sim \\
\hline $\begin{array}{l}\text { Conexão com a } \\
\text { natureza }(\mathrm{CN})\end{array}$ & $3,50 \pm 0,5$ & $3,67 \pm 0,35$ & 1866,5 & 0,15 & Não \\
\hline $\begin{array}{l}\text { Legenda: }{ }^{*} \text { Praça } \mathrm{Ge} \\
\text { Almeidão/Ronaldão e } \\
\text { Branco)/ }{ }^{*} ; * * \mathrm{M}_{\mathrm{d}} \text { (Mec } \\
\text { abdominal (homens) e }\end{array}$ & $\begin{array}{l}\text { ral Lavaner } \\
\text { que da Lago } \\
\text { na) } \pm \text { IIR (int } \\
\text { rras cutâneas }\end{array}$ & Nanderley, & $\begin{array}{l}\text { P Paz, } \\
\text { sto de } 1 \\
\text { * dobra } \\
\text { escapul }\end{array}$ & $\begin{array}{l}\text { Coqueir } \\
\text { é (Praias } \\
\text { s tricipita } \\
\text { res). }\end{array}$ & $\begin{array}{l}\text { Entorno do } \\
\text { Tambaú/Cabo } \\
\text { supra ilíaca e }\end{array}$ \\
\hline
\end{tabular}

Pelo teste de correlação de Spearman observou-se correlações negativas entre aflição e bem-estar $(-0,267726)$, fadiga e bem-estar $(-0,364809)$, afetos negativos e conexão com a natureza $(-0,252734)$, afetos negativos e afetos positivos $(-0,285141)$, RCQ e IMC(-0,475359), dobras cutâneas e RCQ (-0,450685); já as correlações positivas foram entre fadiga e aflição $(0,274839)$, afetos positivos e bem estar $(0,360622)$, afetos negativos e aflição $(0,175612)$, afetos negativos e fadiga $(0,227824)$, IMC e aflição $(0,144842)$ e dobras cutâneas e IMC $(0,563214)$ como pode ser visualizado na Tabela 4 . 
Tabela 4. Matriz de correlação entre as variáveis de composição corporal (IMC, RCQ, dobras cutâneas), da experiência subjetiva com exercício (fadiga, aflição ou mal estar psicológico e bem estar), afeto/emoções (positivos e negativos) e conexão com a Natureza dos entrevistados na pesquisa de campo $(\mathrm{N}=300)$ referentes realizada) da cidade de João Pessoa 2017/2018. Teste de Sperman $(\mathrm{P}<0.05)$.

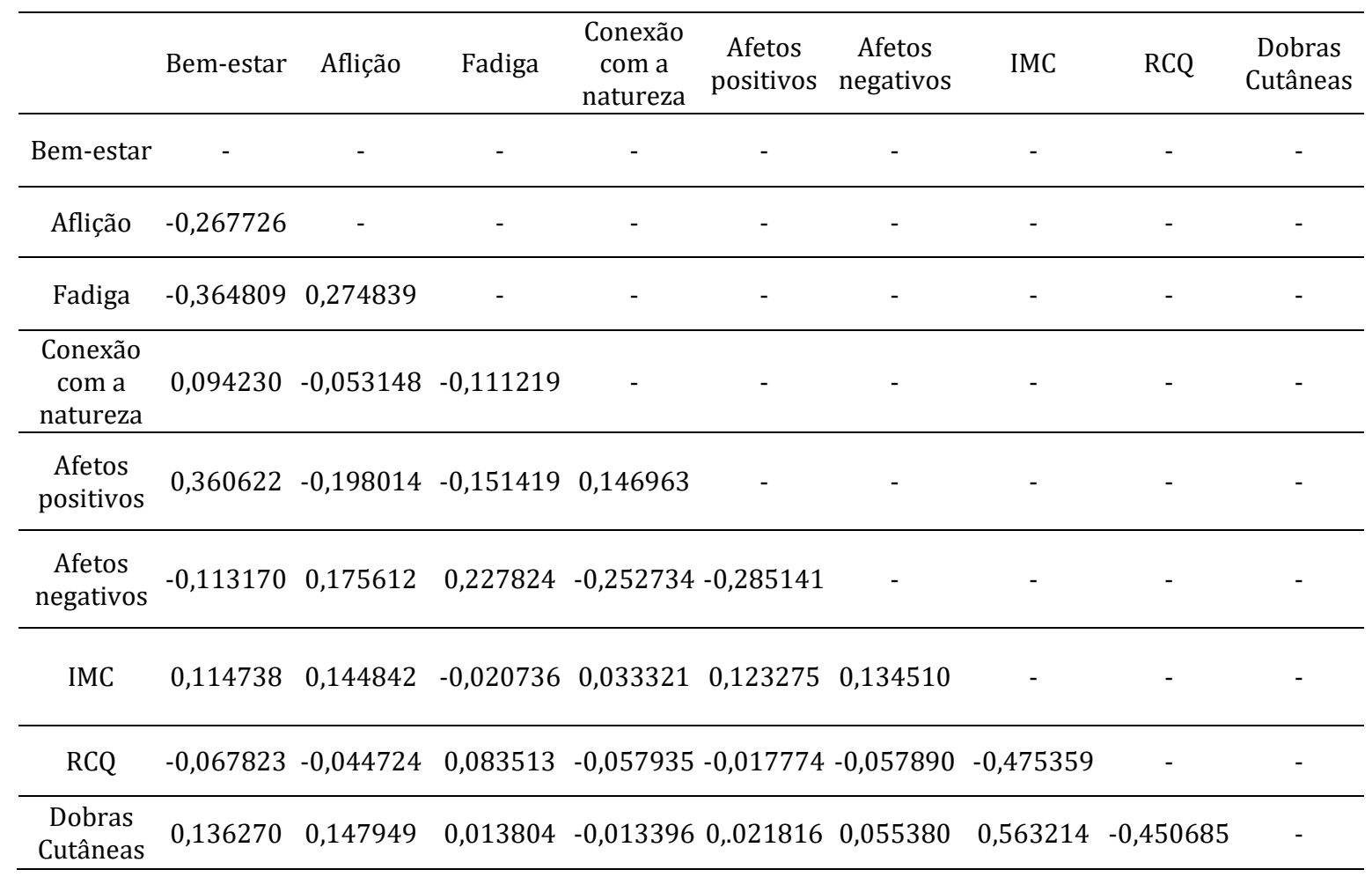

\section{Discussão}

De acordo com os resultados desta pesquisa, não se evidenciou diferenças significativas em termos socioeconômicos (sexo, idade, escolaridade, rendimento salarial e condições de trabalho), no entanto evidenciou-se nesta pesquisa índices maiores de escolaridade e renda/condições de trabalho entre as pessoas com maior idade cronológica (adultos jovens), sendo este comportamento observado em outros estudos envolvendo a prática de exercício regular com adultos (Florindo et al., 2001; Monteiro et al., 1998; Freire e Lélis, 2014).

Um aspecto importante ainda a se destacar na avaliação dos aspectos socioeconômicos é que frequentemente tem-se observado um decréscimo do nível de atividade fisica com o aumento da idade cronológica, sendo recomendado a implantação de políticas públicas de estimulo á prática de exercícios regulares com adultos e idosos, especialmente as mulheres e as pessoas com menor nível de escolaridade (Freire e Lélis, 2014).

Em termos de composição corporal, os resultados desta pesquisa demonstram não existirem diferenças significativas entre o Grupo Outdoor e Grupo Indoor, apenas destacando diferenças com o somatório das dobras cutâneas e com o percentual de gordura corporal (Tabela 2), apontando índices maiores destas variáveis para o grupo outdoor, elemento este provavelmente um indicador que os exercícios físicos indoors desta pesquisa (musculação, ginastica aeróbica/localizada e natação) a nível fisiológico e de composição 
corporal tivessem maior efetividade que os exercícios físicos outdoor desta pesquisa (caminhada, corrida e ciclismo).

Estes resultados apontam dados novos para a literatura da área de saúde e exercício físico, porque alguns estudos apontam resultados similares ou próximo em termos fisiológicos tanto com treinamento com exercícios outdoor, quanto em treinamentos com exercícios indoor, como por exemplo nas melhorias da frequência cardíaca, pressão arterial (Pasanen et al.,2014; Puett et al., 2014; Wood et al., 2016), composição corporal, índice de massa corporal e $\mathrm{VO} 2_{\max }$ (Wood et al., 2016; Lacharité-Lemieux e Dionne, 2016; Krinski et al.,2017) demonstrando assim em termos fisiológicos não existirem diferenças estatisticamente significativas entre as duas formas de treinamento.

Em relação ao bem estar subjetivo pela percepção subjetiva com exercício os resultados apontam diferenças significativas entre o grupo outdoor e indoor no tocante ao bemestar e a fadiga, indicando que as pessoas que praticam exercício ao ar livre (exercício outdoor) possuem índices bem significativos de bem estar subjetivo em relação a pessoas que praticam exercício em ambiente fechados (Grupo Outdoor:6 $\pm 1,25$ / Grupo Indoor: $5 \pm 1,68$,com valores de $\mathrm{W}=72415$ e $\mathrm{p} \leq$ 0,001 ), no entanto observou-se que os indivíduos do Grupo Indoor apresentaram maiores índices de fadiga psicológica que o grupo de indivíduos outdoor (Grupo Outdoor: 2 $\pm 1,5 /$ Grupo Indoor: $3 \pm 2$, com valores de $W=15062$ e $p=<0,001$ ), não se observado diferenças significativas em relação ao parâmetro aflição ou mal estar psicológico, ou seja, o exercício não representa para nenhum dos grupos estado de aflição ou mal estar psicológico, como pode ser visualizado na Tabela 2.

No tocante ao bem estar pelos afetos positivos e negativos, os resultados apontam diferenças estatistas significativas em ambos os parâmetros, sendo que o Grupo Outdoor apresentou maiores índices de afetos positivos que o Grupo Indoor (Grupo Outdoor: $3 \pm 0,5 /$ Grupo Indoor: $3 \pm 0,67, W=73790$ e $\mathrm{p}<0,001$ ), também evidenciou-se no Grupo Indoor maiores índices de afetos negativos que o Grupo Outdoor (Grupo Outdoor: $2 \pm 0,9 /$ Grupo Indoor: $2 \pm 0,8$ ), com valores de $\mathrm{W}=13216$ e $\mathrm{p}<0,001$ ). $\mathrm{Ou}$ seja enquanto o Grupo Outdoor apresenta maiores índices de afetos positivos o Grupo Indoor apresentou maiores índices de aspectos negativos, evidenciando o exercício ao ar livre como um diferencial no tocante aos afetos /emoções relacionados ao bem estar subjetivo.

Estes resultados da pesquisa encontra consonância com outros estudos sobre bem estar apontando para ganhos mais significativos nos aspectos psicoemocionais na prática com exercício outdoor que a prática com exercícios indoor, indicando que os praticantes de exercícios outdoor apresentam melhorias estatisticamente significativas nos afetos positivos, na diminuição dos afetos negativos, na melhoria do bem estar psicológico (Matsouka et al., 2005; Loureiro e Veloso, 2014; Puett et al., 2014), na melhoria do humor (Wood et al., 2016; Niedermeier et al., 2017; Fuegen e Breitenbecher, 2018) e na melhoria da atenção (Weng e Chiang, 2014; Rogerson et al., 2016; Fuegen e Breitenbecher, 2018), demonstrando ainda associação direta no melhoria do foco, atenção afetos positivos, sensação de prazer, humor, energia, adesão ao exercício e diminuição de fadiga (Loureiro e Veloso, 2014; Pasanen et al.,2014; Puett et al., 2014;Weng e Chiang, 2014; Calogiuri et al., 2015; Lacharité-Lemieux e Dionne, 2016; Rogerson et al., 2016; Wood et al., 2016; Krinski et al., 2017; Niedermeier et al., 2017; Fuegen e Breitenbecher, 2018), sendo apenas observado em um estudo que mostra resultados similares de bem estar psicológico com exercício indoor/ outdoor (Matsouka et al., 2005).

Em outro estudo foi observado ainda uma ligação do exercício outdoor 
com o controle do estresse, com melhor perspectiva de vida e melhor percepção de saúde em populações mais ativas, enquanto que o exercício físico indoor pode ser mais associado com as populações pouco ativas (Puett et al., 2014).

Um outro resultado importante encontrado nesta pesquisa foi em relação a conexão com o meio ambiente, encontrando-se diferenças significativas entre o Grupo Outdoor e Grupo Indoor, sendo o Grupo Outdoor apontando uma maior conexão com o meio ambiente que o Grupo Indoor (Grupo Outdoor: $3,57 \pm 0,50$ /Grupo Indoor: $3,35 \pm 0,98$, com valores de $\mathrm{W}=6488$ e $\mathrm{p}<0,001$ ).

Em se tratando da análise de conectividade com o meio ambiente os resultados da pesquisa confirmam a linha de pensamento da maior arte dos estudos envolvendo exercício físico e conexão com o meio ambiente ou natureza, apontando para melhorias na qualidade de vida com a prática de exercício regular ao ar livre (Matsouka et al., 2005; Loureiro e Veloso, 2014; Pasanen et al.,2014; Puett et al., 2014; Weng e Chiang, 2014; Calogiuri et al., 2015; Lacharité-Lemieux e Dionne, 2016; Rogerson et al., 2016; Wood et al., 2016; Krinski et al., 2017; Niedermeier et al., 2017; Araújo e Araújo, 2018; Fuegen e Breitenbecher, 2018), exceto em pacientes com sintomas depressivos, que não apontam melhorias entre treinamento indoor e outdoor (Legrand et al., 2018) e um estudo mostrando que a conectividade com a natureza é um preditor de bem estar subjetivo quando associado ao exercício ao ar livre (Loureiro e Veloso, 2014).

Alguns estudos tem indicado que dentre os treinamentos com exercícios ao ar livre as práticas corporais mais comuns de exercitação física são a caminhada, trilhas, voleibol, ciclismo e tênis, fato esse comprovado em um aumento visível destes praticantes de exercícios regulares nos ambientes urbanos e rurais do Brasil e do mundo de uma forma geral (Matsouka et al., 2005;
Pasanen et al.,2014; Puett et al., 2014; Loureiro e Veloso, 2014; Weng e Chiang, 2014; Calogiuri et al., 2015; LacharitéLemieux e Dionne, 2016; Rogerson et al., 2016; Wood et al., 2016; Krinski et al., 2017; Niedermeier et al., 2017; Fuegen e Breitenbecher, 2018; Legrand et al., 2018).

Também foi feita uma comparação entre os indivíduos do grupo outdoor da praia com os indivíduos das praças, encontrando apenas diferenças significativas entre esses indivíduos no tocante aos afetos negativos, sendo o grupo das praças com maiores índices nos afetos negativos que o grupo das praias (Grupo Outdoor-praças: $2 \pm 0,7$ /Grupo Outdoor-praias: $2 \pm 0,7$, com valores de $W=29395$ e $p<0,001)$, acreditando-se esta diferença está relacionada aos indivíduos das praças serem mais idosos ou com rendas mais baixas que os da praias, podendo estar associada a certas mudanças estruturais do corpo determinadas pelo envelhecimento com implicações psicossociais que acarretam diferentes comportamentos, como inatividade, solidão, isolamento e preconceito, podendo ter essas características afetando um pouco os afetos/emoções negativos destes indivíduos (Freire e Lélis, 2014).

Pelo teste de correlação de Spearman observou-se correlações negativas entre aflição e bem-estar (0,267726), fadiga e bem-estar (0,364809), afetos negativos e conexão com a natureza $(-0,252734)$, afetos negativos e afetos positivos $(-0,285141)$, RCQ e IMC $(-0,475359)$, dobras cutâneas e RCQ (-0,450685 ); já as correlações positivas foram entre fadiga e aflição $(0,274839)$, afetos positivos e bem estar $(0,360622)$, afetos negativos e aflição $(0,175612)$, afetos negativos e fadiga $(0,227824)$, IMC e aflição $(0,144842)$ e dobras cutâneas e IMC $(0,563214)$ como pode ser visualizado na Tabela 4 .

0 teste de correlação de Spearman vem corroborar os resultados do teste de Mann-Whitney, indicando 
pelas correlações negativas enquanto menor aflição maior bem estar, menor fadiga maior bem estar, menor afetos negativos maior conexão com a natureza, menor afetos negativos maior afetos positivos, menor RCQ maior IMC, menor dobras cutâneas maior RCQ; Já pelas correlações positivas enquanto maior afetos positivos maior bem estra, maior fadiga maior aflição, maior afetos negativos maior aflição, maior afetos negativo maior fadiga, maior IMC maior aflição e maior dobras cutâneas maior IMC, como pode ser visualizado na Tabela 4.

\section{Conclusões}

Estes resultados demonstram as relações existentes entre exercício outdoor/indoor, meio ambiente, bem estar e saúde, sendo um parâmetro importante para o fortalecimento $\mathrm{e}$ ampliação dos programas de treinamento com exercícios físicos para a melhoria e manutenção da saúde e qualidade de vida da população.

Futuros estudos precisam aprofundar estas analises com um número maior de indivíduos e ampliar as análises sobre estas melhorias já demonstradas, buscando assim subsídios importantes do fortalecimento dos programas de exercícios físicos regulares tanto outdoor como indoor para a promoção da saúde e qualidade de vida da população.

\section{Conflito de interesses}

Os autores declaram não haver conflito de interesses.

\section{Referências}

Albuquerque, A. S.; Tróccoli, B. T. Desenvolvimento de uma escala de bem-estar subjetivo. Psicologia: Teoria e Pesquisa, v. 20, n. 2, p. 153-164, 2004. https://doi.org/ 10.1590/S0102-37722004000200008

Alves, H. N.; Fridich, G. A.; Souza, T. S. P.; López, L. C. S.; Lucena, R. F. P. Exercício físico outdoor e indoor, bem-estar subjetivo e conexão com a Natureza: uma revisão sistemática. Revista Brasileira de Gestão Ambiental e Sustentabilidade, v. 6, n. 13, p. 515-529, 2019. https://doi.org/10.21438/ rbgas.061319

Araújo, D. S. M.; Araújo, C. G. S. Aptidão física, saúde e qualidade de vida relacionada à saúde em adultos. Revista Brasileira de Medicina do Esporte, n. 6, v. 5, p. 194-203, $2000 . \quad$ https://doi.org/10.1590/S151786922000000500005

Brasil. Conselho Nacional de Saúde. Resolução no 466, de 12 de dezembro de 2012. Disponível em: <https://conselho. saude.gov.br/resolucoes/2012/Reso466.pdf >. Acesso em: 23 abr. 2019.

Cabral, A.; Palmeira, A. Validação preliminar da escala da experiência subjetiva ao exercício - EESE, para a língua portuguesa. In: Malico, P.; Antunes, J; Palmeira, A. Actas das IX Jornadas Nacionais de Psicologia do Desporto, 2003.

Calogiuri, G.; Nordtug, H.; Weydahl, A. The potential of using exercise in nature as an intervention to enhance exercise behavior: Results from a pilot study. Percept Motor Skills, v. 121, n. 2, p. 350-370, 2015. https://doi.org/10.2466/06.PMS.121c17x0

Carvalho, Y. M. Atividade física e saúde: onde está e quem é o "sujeito" da relação? Revista Brasileira de Ciências do Esporte, v. 22, n. 2, p. 9-21, 2001.

Dehoog, S. Avaliação do estado nutricional. In: Mahan, L. K.; Krause, M. V. Alimentos, nutrição e dietoterapia. 9. ed. São Paulo: Rocca, 1998. p. 371-395.

Diener, E.; Napa Scollon, C.; Lucas, R. E. The evolving concept of subjective well-being: The multifaceted nature of happiness. In: Diener E. (Eds.). Assessing well-being. Dordrecht: Springer, 2009. (Social Indicators Research Series, v. 39). https://doi.org/ 10.1007/978-90-481-2354-4_4

Florindo, A. A.; Latorre, M. R. D.; Tanaka, T.; Jaime, P. C.; Zerbini, C. A. F. Fatores associados à prática de exercícios físicos em homens voluntários adultos e idosos residentes na Grande São Paulo, Brasil. Revista Brasileira de Epidemiologia, v. 4, n. 2, p. 105-113, 2001. https://doi.org/ 10.1590/S1415-790X2001000200005 
Florindo, A. A.; Salvador, E.; Reis, R. S.; Guimarães, V. V. Percepção do ambiente e prática de atividade física em adultos residentes em região de baixo nível socioeconômico. Revista de Saúde Pública, v. 45, n. 2, p. 302-310, 2011. https://doi.org/ $10.1590 /$ S0034-89102011000200009

Freire, R. F.; Lélis, F. L. O. Prática regular de atividade física: estudo de base populacional no norte de Minas Gerais, Brasil. Revista Brasileira de Medicina do Esporte, v. 20, n. 5 , p. 345-349, 2014. https://doi.org/ 10.1590/1517-86922014200502062

Fuegen, K.; Breitenbecher, K. H. Walking and being outdoors in nature increase positive affect and energy. Ecopsychology, v. 10, n. 1, p. 14-25, 2018. https://doi.org/10.1089/ eco.2017.0036

Garcez, A. R. M. Relatório de estágio da prática de ensino supervisionada. Guarda, Portugal: Escola Superior de Educação, Comunicação e Desporto Instituto Politécnico da Guarda, 2017. (Dissertação de mestrado).

Garrow, J. S. Indices of adiposity. Nutrition Abstracts and Reviews - Series A, v. 53, n. 8, p. 697-708, 1983.

Graciano, M. I. G. Estudo socioeconômico: um instrumento técnico-operativo. Bauru: Universidade de São Paulo, Hospital de Reabilitação de Anomalias Craniofaciais, 2013. (Curso de Anomalias Congênitas Labiopalatinas).

Guedes D. P. Composição: princípios, técnicas e aplicações. 2. ed. Londrina: APEF, 1994.

Haskell, W. L.; Lee, I. M.; Pate, R. R.; Powell, K.; Blair, S.; Franklin, B.; Macera, C.; Heath, G.; Thompson, P.; Bauman, A. Physical activity and public health: Updated recommendation for adults from the American College of Sports Medicine and the American Heart Association. Medicine \& Science in Sports \& Exercise, v. 116, n. 9, p. 1081-1093, 2007. https://doi.org/10.1249/mss.0b013e318061 $6 \mathrm{~b} 27$

Krinski, K.; Machado, D. G. S.; Lirani, L. S.; Silva, S. G.; Costa, E. C.; Hardcastle, S. J., Elsangedy, H. M. Let's walk outdoors! Self paced walking outdoors improves future intention to exercise in women with obesity. Journal of Sport and Exercise Psychology, v. 39, n. 2, p. 145-157, 2017. https://doi.org/ 10.1123/jsep.2016-0220
Lacharité-Lemieux, M.; Dionne, I. J. Physiological responses to indoor versus outdoor training in postmenopausal women. Journal of Aging and Physical Activity, v. 24, n. 2, 275-283, 2016. https://doi.org/ 10.1123/japa.2015-0019

Legrand, F. D.; Race, M.; Herring, M. P. Acute effects of outdoor and indoor exercise on feelings of energy and fatigue in people with depressive symptoms. Journal of Environmental Psychology, v. 56, n. 4, p. 91-96, 2018. https://doi.org/10.1016/ j.jenvp.2018.03.005

Loureiro, A.; Veloso, S. Outdoor exercise, well-being and connectedness to nature. Psico, v. 45, n. 3, p. 299-304, 2014.

Marselle, M. R.; Irvine, K. N.; Warber, S. Examining group walks in nature and multiple aspects of well-being: A large scale study. Ecopsychology, v. 6, n. 3, p. 134-148, 2014. http://doi.org/10.1089/eco.2014.0027

Matsouka, O.; Kabitsis, C.; Harahousou, Y.; Trigonis, I. Mood alterations following an indoor and outdoor exercise program in healthy elderly women. Perceptual and Motor Skills, v. 100, n. 3, 707-715, 2005. http://doi.org/10.2466/PMS.100.3.707-715

Mayer, F. S.; Franz, C. M. The connectedness to nature scale: A measure of individuals' feeling in community with nature. Journal of Environmental Psychology, v. 24, n. 4, p. 503-515, 2004. http://doi.org/10.1016/ j.jenvp.2004.10.001

Minayo, M. C. S.; Hartz, Z. M. A.; Buss, P. M. Qualidade de vida e saúde: um debate necessário. Ciências \& Saúde Coletiva, v. 5, n. 1, p. 7-18, 2000. https://doi.org/10.1590/ S1413-81232000000100002

Monteiro, H. L.; Gonçalves, A.; Padovani, C. R.; Fermino Neto, J. L. Fatores socioeconômicos e ocupacionais e a prática de atividade física regular: estudo a partir de policiais militares em Bauru, São Paulo. Motriz, v. 4, n. 2, p. 9297, 1998. https://doi.org/10.5016/6609

Nahas, M. V.; Barros, M. V. G.; Francalacci, V. 0 pentáculo do bem-estar: base conceitual para avaliação do estilo de vida de indivíduos ou grupos. Revista Brasileira de Atividade Física \& Saúde, v. 5, n. 2, p. 48- 59, 2000. https://doi.org/10.12820/rbafs.v.5n2p48-59

Niedermeier, M.; Einwanger, J.; Hartl, A.; Kopp, M. Affective responses in mountain 
hiking: A randomized crossover trial focusing on differences between indoor and outdoor activity. PLoS ONE, v. 12, n. 5, p. 1-17, 2017. https://doi.org/10.1371/journal.pone.01777 19

Pasanen, T. P; Tyrväinen, L.; Korpela, K. M. The relationship between perceived health and physical activity indoors, outdoors in built environments and outdoors in nature. Applied Psychology: Health and WellBeing, v. 6, n. 3, p. 324-346, 2014. https://doi.org/10.1111/aphw.12031

Pessoa, V. S.; Gouveia, V. V.; Soares, A. K. S; Vilar, R.; Freires, L. A. Escala de conexão com a natureza: evidências psicométricas no contexto brasileiro. Estudos de Psicologia, v. 33, n. 2, p. 271-282, 2016. https://doi.org/ 10.1590/1982-02752016000200009

Petroski, E. L. Equações antropométrica: subsídios para uso do estudo da composição corporal. In: Petroski, E. L. Antropometria: técnica e padronização. Porto Alegre: Palotti, 1999. p. 69-86.

Piko, B. F.; Keresztes, N. Physical activity, psychosocial health, and life goals among youth. Journal of Community Health, v. 31, n. 2, p. 136-145, 2006. https://doi.org/ 10.1007/s10900-005-9004-2

Pollock, M. L.; Wilmore, J. H. Exercícios na saúde e na doença: avaliação e prescrição para prevenção e reabilitação. São Paulo: MEDSI, 1993.

Puett, R.; Teas, J.; España-Romero, V.; Artero, E. G.; Lee, D. C.; Baruth, M.; Sui, X.; MontresorLópez, J.; Blair, S. N. Physical activity: Does environment make a difference for tension, stress, emotional outlook, and perceptions of health status? Journal of Physical Activity \&
Health, v. 11, n. 8, p. 1503-1511, 2014. https://doi.org/10.1123/jpah.2012-0375

Rogerson, M.; Gladwell, V. F.; Gallagher, D. J.; Barton, J. L. Influences of green outdoors versus indoors environmental settings on psychological and social outcomes of controlled exercise. International Journal of Environmental Research and Public Health, v. 13, n. 4, p. 363-378, 2016. https://doi.org/10.3390/ijerph13040363

Silva, I. J. O.; Alexandre, M. G.; Ravagnani, F. C. P.; Silva, J. V. P.; Coelho-Ravagnani, C. F. Atividade física: espaços e condições ambientais para sua prática em uma capital brasileira. Revista Brasileira de Ciência e Movimento, v. 22, n. 3, p. 53-62, 2014.

Siqueira, M. M. M.; Padovan, V. A. R. Bases teóricas de bem-estar subjetivo, bem-estar psicológico e bem-estar no trabalho. Psicologia: Teoria e Pesquisa, v. 24, n. 2, p. 201-209, 2008. https://doi.org/10.1590/ S0102-37722008000200010

Watson, D.; Clark, L. A. The PANAS-X: Manual for the positive and negative affect scheduleexpanded form. Ames: The University of Iowa, 1994. https://doi.org/10.17077/48vt$\mathrm{m} 4 \mathrm{t} 2$

Weng, P. Y.; Chiang, Y. C. Psychological Restoration through Indoor and Outdoor Leisure Activities. Journal of Leisure Research, v. 46, n. 2, p. 203-217, 2014. https://doi.org/10.1080/00222216.2014.11 950320

Wood, C. J.; Pretty, J.; Griffin, M. A casecontrol study of the health and well-being benefits of allotment gardening. Journal of Public Health, v. 38, n. 3, p. 336-344, 2016. 